\title{
Condutas desviantes e traços de personalidade: testagem de um modelo causal ${ }^{1}$
}

\author{
Deviant behavior and personality traits: \\ testing of a causal model
}

\author{
Tatiana Cristina VASCONCELOS² \\ Valdiney Veloso GOUVEIA ${ }^{3}$ \\ Carlos Eduardo PIMENTEL ${ }^{3}$ \\ Viviany Silva PESSOA ${ }^{3}$
}

\begin{abstract}
Resumo
A meta principal deste estudo foi comprovar a adequação de um modelo causal à explicação de comportamentos socialmente desviantes (condutas anti-sociais e delitivas), considerando a contribuição dos traços de personalidade (neuroticismo, extroversão e busca desensações). Para tanto, participaram 755 estudantes do Ensino Médio e Superior, sendo a maioria do sexo feminino $(50,3 \%)$, de escolas privadas (53,0\%) e com idades variando de 16 a 26 anos (média=20,1; desvio-padrão=3,12). Estes responderam ao Inventário dos Cinco Grandes Fatores de Personalidade, à Escala de Busca de Sensações e ao Questionário de Condutas Anti-Sociais e Delitivas. Os principais resultados indicaram que os traços neuroticismo e busca de sensações explicam satisfatoriamente as condutas anti-sociais, e estas predizem diretamente as condutas delitivas. Neste aspecto, conseguiu-se constatar que os traços de personalidade são úteis ao entendimento das condutas socialmente desviantes, com especial destaque para o traço busca desensações. Destaca-se, no entanto, a necessidade de estudos posteriores considerando outras variáveis e contextos. Unitermos: Busca de sensação. Extroversão. Transtorno da personalidade anti-social.
\end{abstract}

\begin{abstract}
The main purpose of thisstudy wasto test theadequacy of a causal model to explain socially deviant behavior (i.e. antisocial and criminal behavior), taking into account the contribution of personality traits (neuroticism, extraversion, and sensation seeking). To thisend, 755 high school and undergraduate students participated, most of them female (50.3\%) and from private schools/universities (53\%), with ages ranging from 16 to 26 (average=20.1; standard deviation=3.12). They answered a questionnaire in which three different measures were incorporated:Big Five Inventory, Sensation Seeking Scale, and Antisocial and Criminal Behavior Questionnaire. The main results demonstrated that the traits of neuroticism and sensation-seeking satisfactorily explained the antisocial behavior, and these directly predicated criminal behavior. It was confirmed that the personality traits, especially sensation seeking, are helpful in the understanding of socially deviant behavior. However, the need for further studies incorporating other variablesand contexts should beemphasized.

Uniterms: Sensation seeking. Extraversion. Antisocial personality disorder.
\end{abstract}

\footnotetext{
${ }^{1}$ Artigo elaborado a partir da dissertação de T.C.VASCONCELOS, intitulada "Personalidade, valores e condutas anti-sociais de jovens". Universidade Federal da Paraíba, João Pessoa, 2004.

2 Faculdade Santa Maria. Cajazeiras, PB, Brasil.

3 Universidade Federal da Paraíba, Centro de Ciências Humanas, Letras e Artes, Departamento de Psicologia. Campus I, Cidade Universitária, s/n., 58051-900, J oão Pessoa, PB, Brasil. Correspondência para/Correspondence to: V.V.GOUVEIA. E-mail: <vvgouveia@gmail.com>.

Agradecimentos à Coordenação de Aperfeiçoamento de Pessoal de Nível Superior e ao Conselho Nacional de Desenvolvimento Científico e Tecnológico, pelo apoio financeiro para realização desta pesquisa, em forma de bolsa de mestrado para a primeira autora e de produtividade para o segundo.
} 
A sociedade atualmente vive um contexto em que adolescentes e jovens apresentam comportamentos anti-sociais e violentos, cada dia mais freqüentes. Tal aumento é confirmado por diversos estudos (Adalbjarnardottir \& Rafnsson, 2002; Ding, Nelsen \& Lassonde, 2002; Engels \& Bogt, 2001; Hawkins, Catalano \& Miller, 1992; Herrenkohl et al., 2000; Patterson, DeBaryshe \& Ramsey, 1989; Saner \& Ellickson, 1996), caracterizando-se como um fenômeno social e de saúde pública. Diante deste fato, surgem questões do tipo: quais os motivos que levam alguns jovens a se envolverem em situações que rompem as normas sociais, bem como em situações violentas? $\mathrm{E}$, ainda, porque alguns jovens se envolvem em tais situações, enquanto outros não o fazem? Constata-se que estas, assim como outras questões, não são fáceis de serem respondidas, pois a complexidade e multideterminação do comportamento humano exigem uma análise cautelosa na sua explicação.

Pode-se dizer que os jovens e adolescentes parecem ser naturalmente mais suscetíveis a transgredir normas e regras sociais (Coelho Júnior, 2001). Pesquisas estadunidenses apontam que as pessoas nesta fase de desenvolvimento são mais prováveis causadoras ou vítimas de violência interpessoal, em comparação a indivíduos adultos (Herrenkohl et al., 2000). No caso do Brasil, Waiselfisz (2002) menciona que, no conjunto da população, $12,2 \%$ do total de mortes são atribuíveis a causas exógenas (homicídios, acidentes de automóveis). Entre os jovens, as mesmas são responsáveis por mais de $70,0 \%$ dos óbitos.

Na população brasileira, 4,7\% das mortes são devidos a homicídios e, quando considerados especificamente entre os jovens, esses são responsáveis por $39,2 \%$ das mortes. Segundo Waiselfisz (2002), é na faixa dos 15 aos 24 anos que os homicídios atingem sua maior incidência, e o momento crítico, com maior risco de ser vítima de homicídio, é na idade de 20 anos. Nos últimos dez anos, percebe-se que houve um aumento de 77,0\% no número de vítimasjovens, sendo o Nordeste a região que apresentou o maior crescimento no índice $(60,7 \%)$, seguido pelo Sudeste $(55,8 \%)$ e Centro-Oeste (55,9\%).

Parece plausível falar em um aumento epidêmico dos fenômenos violentos e delinqüentes na sociedade, e isso tem sido enfocado constantemente pela mídia. Diferentes jornais e noticiários televisivos apresentam, quase diariamente, variadas formas de expressão das condutas violentas e anti-sociais (Formiga, 2002; Gouveia, Coelho Júnior, Gontiès, Andrade \&Andrade, 2003). Estudar seus antecedentes deveria ser prioritário, pois permitiria, por exemplo, desenvolver políticas e delinear programas de prevenção e controle de alguns problemas sérios que the são correlatos durante a adolescência, a exemplo do uso/envolvimento com drogas (Coelho Júnior, 2001; Romero, Luengo \& Sobral, 2001).

A partir desta problemática, a presente pesquisa buscou comprovar, pela adequação de um modelo causal, a influência dos traços de personalidade na explicação das condutas anti-sociais e delitivas, com destaque para o modelo dos Big Five (Cinco Grandes Fatores de Personalidade) e do traço busca desensações. No decorrer deste texto, são apresentadas considerações mais detalhadas sobre tais construtos.

\section{Condutas desviantes: anti-sociais e delitivas}

As condutas dos adolescentes e jovens, por possuírem aspectos peculiares, são fontes de muitas pesquisas, especialmente das que consideram aspectos anti-sociais. Ainda mais porque, nos dias atuais, vê-se que os episódios de adolescentes e jovens envolvidos com drogas, roubos e até homicídios têm sido amplamente salientados.

Hawkins et al. (1992) apresentam uma gama de estudos que abordam a violência juvenil, a delinqüência, as condutas anti-sociais, bem como os sinônimos e as facetas deste problema social. Cabe, então, especificar que o termo condutasanti-sociaisfaz referência a todas as condutas consideradas socialmente indesejáveis, que podem afetar o bem-estar social, enquanto as condutas delitivasrepresentam uma violação à lei (Formiga, 2002; Pimentel, 2004; Scaramella, Conger, Spoth $\&$ Simons, 2002), tendo como precedentes as condutas anti-sociais.

A respeito da relação entre condutas anti-sociais e delitivas, cabe esclarecer que toda conduta delitiva é anti-social, contudo, nem sempre o contrário é verdadeiro. 0 que as condutas anti-sociais e delitivas têm em comum é que ambas interferem nos direitos e deveres das pessoas, ameaçando o seu bem-estar (Formiga, 2002). Como faz parte do repertório do jovem o desafio 
aos padrões tradicionais da sociedade,é possível afirmar que a maioria destes pratica ou já praticou algum tipo de conduta anti-social, no sentido amplo (Coelho Júnior, 2001). No presente artigo, condutas desviantes, portanto, faz referência aos dois tipos de condutas antes discutidas.

Segundo Pfromm Neto (1979), os adolescentes que ap resentam condutas delinqüentes (ou desviantes) compreendem um foco de grande preocupação na sociedade, por se tornarem indesejáveis. Tais jovens, violadores de normas e leis, caracterizam-se por apresentar uma personalidade que prioriza valores que vão de encontro às normas sociais e à orientação cultural (Formiga, 2002).

É importante expor, ainda, que a análise de tais condutas, ditas socialmente indesejáveis, está vinculada a três elementos indisso ciáveis, quais sejam: o comportamento em si, o indivíduo que o adota e o ambiente sócio-cultural. Em outras palavras, expõe-se aqui a idéia de que nem tudo que é anti-social em uma sociedade e contexto histórico será em outros.

As condutas, apesar de serem vistas como dinâmicas, funcionais, podendo ser compreendidas em função do contexto em que ocorrem, geralmente são consideradas como expressões da personalidade dos indivíduos, e podem ser explicadas em termos de traços de personalidade (Paunonen \& Ashton, 2001). Não obstante, essa é uma hipótese que precisa ser comprovada. Cabe, neste instante, conhecer mais acerca da personalidade tal como foi tratada neste estudo.

\section{Personalidade}

A análise sistemática da personalidade é historicamente um tópico de grande relevância no âmbito da psicologia, tendo, nosúltimos anos, recebido especial destaque a discussão acerca das suas dimensões principais (Benet-Martínez \& John, 1998). No entanto, a literatura brasileira acerca desta temática ainda é escassa, especificamente no que diz respeito à sua análise empírica(Hutzet al. 1998; J esus, 2001; Queiroga, 2002).Ademais, dentre os estudos empíricos que vêm sendo realizados, poucos são os que a consideram em relação a outros construtos, como as condutas anti-sociais e pró-sociais.

Diferentes abordagens dedicaram-se ao estudo da personalidade (Allport, 1973; Hall, Lindzey \&Campbell,
2000; Pervin, 1978). Dentre as significativas contribuições, encontram-se os escritos de Allport (1973), que advogou que o nível mais proveitoso de estudo para a personalidade era o traço, dando origem a uma nova maneira de refletir acerca deste construto. Allport considerava traço de personalidade como sendo predisposições a responder igualmente ou de um modo semelhante a tipos diferentes de estímulos, ou seja, formas constantes e duradouras de reagir ao ambiente. Suas características seriam de individualidade, natureza real, determinação do comportamento, ser passível de demonstração empírica e possuir variações situacionais e inter-relacionais (Schultz \&Schultz, 2002). Neste sentido, o traço pode ser empregado para resumir como as pessoas são ou se comportam no seu dia-a-dia.

Baseados nesta visão, outros teóricos levaram a cabo estudos sobre a personalidade, focalizando, todavia, aspectos diferentes, como é o caso das teo rias fatoriais. Cattell foi um dos precursores no uso da análise fatorial para o estudo de variáveis múltiplas, propondo o seu modelo dos 16-PF (Dezesseis Fatores de Personalidade), com a finalidade de obter um conjunto consistente de itens capaz de medir objetivamente a personalidade (Cattell \& Cattell, 1995; J esus, 2001; Queiroga, 2002).

Todavia, na época em que Cattell realizou a análise fatorial para elaboraro seu modelo, esta técnica ainda era bastante limitada e o modelo, bastante complexo, o que propiciou várias críticas, como observam Hutz et al. (1998), abrindo caminho para muitos modelos inovadores acerca das dimensões da personalidade, enfatizando diferentes quantidades de fatores.

Dos modelos que vêm sendo propostos, 0 OCEAN (abertura, consciencioso, extroversão, sociabilidade e neuroticismo), elaborado por Costa e McCrae (1992), propõe cinco dimensões da personalidade, baseando-se na análise fatorial de uma série de questionários. 0 Big Five, modelo em que se baseou este trabalho, tem bastante em comum com o PEN (psicoticismo, extroversão, neuroticismo), criado por Eysenck (1990, 1991), e inclusive compartilham os fatores de extroversão e neuroticismo.

O Big Five vem ganhando destaque na literatura sobre a estrutura da personalidade. Ele tem se mostrado adequado, apresentando um poder de síntese satisfatório e contando com alguns estudos a respeito no Brasil (Hutz et al. 1998; Jesus, 2001; Queiroga, 2002), 
evidenciando a idéia de que existem dimensões básicas da personalidade. Por esta razão, foi adotado o modelo do Big Five na análise deste estudo. Justifica-se, então, que este seja também resumido mais detidamente a seguir.

\section{O modelo dos cinco grandes fatores}

No trabalho intitulado Las Cinco Grandes Dimensiones dela Personalidad, de 1996, J an ter Laak faz um apanhado histórico do surgimento do Modelo dos Cinco Grandes Fatores (MCGF) da personalidade, e comenta que as análises de correlação de diversos instrumentos que medem os traços ou disposições geralmente indicam duas dimensões, interpretadas como extroversão e neuroticismo.

As pesquisas correlacionais com enfoque psicoléxico, ou seja, aquelas que utilizam adjetivos de personalidade, levaram a agregar outras três dimensões, a saber: agradabilidade (sociabilidade), escrupulosidade (conscienciosidade) e abertura à mudança (Laak, 1996). Este novo conjunto de dimensões passou a ser conhecido como os Cinco Grandes Fatores de Personalidade (Cattell, 1996; Goldberg, 1981).

Desde a publicação dos primeiros estudos a respeito dos cinco fatores, diversos autores têm se empenhado em abordar esta questão cientificamente, propondo inclusive novos instrumentos para medi-los (Benet-Martínez \&J ohn, 1998; Hofstee, Raad \&Goldberg, 1992).

Falar em um modelo composto por cinco fatores de personalidade significa prover uma linguagem comum para psicólogos de diferentes tradições, um fenômeno básico para os teóricos da personalidade explicarem uma estrutura natural para organizar a pesquisa, e um guia para a avaliação compreensiva dos indivíduos, podendo ser de valor para psicólogos educacionais, industriais/organizacionais e clínicos (Queiroga, 2002). Todavia, mesmo seus mais ardentes defensores não proclamam que o modelo dos cinco fatores é a última palavra na descrição da personalidade, existindo disputas entre os five-fatoristas so bre a mel hor interpretação dos fatores (McCrae \& J ohn, 1992).

Costa e McCrae (1992) e Goldberg (1981) são, provavelmente, os maiores proponentes do MCGF, tendo como embasamento teórico para seus estudos a díade genética/ambiente, e a hipótese léxica. Esta última é defendida como a melhor estratégia para entender as características da personalidade, uma vez que as pessoas tendem a ressaltar na linguagem suas diferenças mais significativas (Goldberg, 1981). Para Goldberg, se uma característica de personalidade for saliente, ou seja, capaz de gerar diferenças individuais socialmente relevantes, as pessoas vão notar esta característica e, por ser importante, vão querer falar sobre ela. Como conseqüência, uma palavra ou expressão possivelmente será criada para descrever essa característica ou traço.

Embora haja diferenciação em relação ao suporte teórico dos itens utilizados por alguns dos autores, observa-se certa convergência acerca das etiquetas para os fatores, podendo os mesmos ser apresentados da seguinte forma: agradabilidade, fator empregado para expressar a tendência de o indivíduo ser socialmente agradável, amigável, caloroso, dócil, ou não, e em que nível isto ocorre (Goldberg, 1981); conscienciosidade, fator também conhecido por escrupulosidade ou vontade de realização (will to achive) (Hutz et al. 1998; Queiroga, 2002), que é, no geral, um fator que agrupa traços ou características de personalidade que apresentam responsabilidade e honestidade, em um extremo, e negligência e irresponsabilidade, no outro;e abertura à mudança, fator também denominado de abertura a experiências e intelecto, que agrupa características como flexibilidade de pensamento, fantasia e imaginação, abertura a experiências novas, principalmente aquelas com um foco cultural (Five Factor Model, 2001; Goldberg, 1981; Hutz et al. 1998).

Os dois últimos dos cinco fatores fazem parte da maioria dos instrumentos de avaliação da personalidade (ver também comentários de Hutz et al. 1998), e são eles: extroversão, fator que sintetiza traços relacionados à atividade e energia, dominância, sociabilidade, expressividade e emoções positivas, ou, ainda, que procura saber se a pessoa é ativa e dominante, ou passiva e submissa (Benet-Martínez \& John, 1998; Goldberg, 1981); e neuroticismo, fator que contrasta emoções estáveis com afetos negativos, incluindo ansiedade, tristeza, irritabilidade e tensão nervosa (Benet-Martínez \&John, 1998) e, resumidamente, caracteriza a estabilidade emocional do sujeito, reunindo características ou traços de personalidade que procuram saber se a pessoaé desequilibrada, imprevisível, ou "equilibrada", estável (Goldberg, 1981). 
Ainda que se comente ser improvável que estes sejam os únicos fatores da personalidade, parece relevante considerar a organização da personalidade em termos de cinco dimensões básicas, sendo estas úteis tanto para a avaliação individual, quanto para auxiliar os psicólogos estudiosos da personalidade a compreender outros fenômenos psíquicos (McCrae \& J ohn, 1992).

Segundo Perugini, Gallucii e Livi (2000), o BigFive pode, atualmente, ser considerado como o melhor candidato para representar o sistema coordenado da estrutura da personalidade. A este respeito, observa-se que um número considerável de pesquisas tem se dedicado a estudar diversos construtos a partir do MCGF. Nestas pesquisas são abordadas, por exemplo, as relações dos diferentes construtos com aspectos culturais, buscando saber acerca das características de personalidade que são universais ou específicas, dos traços em distintas culturas ocidentais e orientais (Digman, 1990; Gouveia, Meira, Santos, Jeans \& Formiga, 2001; Katgbak, Church, Cuazon-Lapefia, Carlota, Del Pilar, 2002), e dos traços em relação às diferenças de gênero e/ou idade (Feingold, 1994; Queiroga, 2001).

\section{Busca de sensações}

Outro traço de personalidade que tem sido estudado é a busca de sensações. Marvin Zuckerman, desde a segunda metade dos anos 1960 até a atualidade, busca analisar e aprimorar a teoria deste traço (Zuckerman, 1994). Este autor verificou que ele se apresenta em todos os indivíduos, variando apenas na intensidade, sendo observadas na faixa-etária que varia entre 16 e 20 anos (ver também Kopeikin, 1997) as suas manifestações mais intensas.

A busca de sensações é definida por Zuckerman (1994, p. 1) como "Um traço que descreve a tendência para procurar sensações e experiências novas variadas, complexas e intensas, e a disposição para correr riscos com a finalidade de satisfazer tais experiências". Esta é apontada como uma característica peculiar ao adolescente, estando, na maioria das vezes, relacionada com comportamentos de risco, como, por exemplo, uso de drogas, atividades de mergulho e de pára-quedismo, além de dirigir embriagado (Lin \& Tsai, 2002). Neste sentido, parece clara a necessidade de considerar tal construto neste estudo.
Dentre algumas características co muns aos chamados altos buscadores de sensações estão as atitudes positivas em relação à emoção ou alegria, e expressões mais desinibidas. Assim, espera-se que a busca de sensações prediga uma abertura à mudança, uma atitude receptiva em relação às novas experiências, e a habilidade para tolerar sensações e idéias que são comumente estranhas para a maioria das pessoas. Os altos buscadores de sensações são também considerados como mais sociáveis, impetuosos, assertivos, atrevidos e demonstram menos medo (Aluja, 1989, 1990; Zuckerman, 1983, 1994; Zuckerman, Eysenck \& Eysenck, 1978).

Para mensurar a busca de sensações, a Escala de Busca de Sensação (EBS), proposta por Marvin Zuckerman, é a mais utilizada. A primeira versão surgiu em 1964, a qual continha um só fator geral, derivado racionalmente, ou seja, tomando como referência a concepção de um construto que deveria expressar. Em função das pesquisas realizadas, tiveram lugar outras versões, compostas por quatro sub-escalas, derivadas de análise fatorial: 1) Busca deAventuraseEmoção (BEM); 2) Busca de Experiências (BEX); 3) Desinibição (DES); e 4) Suscetibilidadeao Aborrecimento (SAB). Em estudos com ingleses e holandeses, corroborou-se a presença destes quatro fatores (BEM, $B E X, D E S, S A B$ ), resultando na versão $\checkmark$ da escala, composta por 10 itens em cada fator.

Greene, Kcrmar, Walters, Rubin e Hale. (2000) observaram que os quatro fatores de busca desensação estão diretamente correlacionados com um conjunto de comportamentos anti-sociais, a exemplo de fumar $(r=0,22, p<0,001)$, prática sexual arriscada $(r=0,15$, $p<0,001)$, uso de drogas $(r=0,37, p<0,001)$, delinqüência $(r=0,38, p<0,001)$, usar bebidas alcoólicas $(r=0,43, p<0,001)$ e dirigir embriagado $(r=0,30, p<0,001)$. Resultados muito similares foram observados por Donohew et al. (1999), no que se refere ao uso de maconha eálcool. Estes mesmos autores reafirmam os achados de Zuckerman (1994), ressaltando que os quatro fatores apresentados podem ser reduzidos a uma só dimensão. Assim, diante do exposto, considera-se que a busca de sensações pode contribuir para entender as condutas anti-sociais e delitivas dos adolescentes e jovens, como também pode ser melhor estudada em relação aos traços de personalidade. 
Condutas desviantes, Big Fivee busca de sensações

Em vários estudos, tentou-se estabelecer a relação entre traços de personalidade e condutas desviantes. Usando o modelo de Hans Eysenck, por exemplo, Romero et al. (2001), em amostras de estudantes espanhóis, encontraram que os fatores neuroticismo e psicoticismo se correlacionaram com condutas anti-sociais $\left(r_{\text {médio }}=0,14\right.$ e 0,32, respectivamente; $p<0,01$ para ambos). Heaven (1994), com estudantes de Ensino Médio da Austrália, observou que o psicoticismo se correlacionou com medidas de vandalismo $\left(r_{\text {médio }}=0,35\right.$, $p<0,001)$ e violência ( $\left.r_{\text {médio }}=0,37, p<0,001\right)$.

No caso do Big Five, Gullone e Moore (2000) afirmam que o padrão de correlação dos traços de personalidade com as condutas anti-sociais depende dos fatores de risco que são considerados. Para eles, os traços de personalidade foram mais eficazes para predizer os comportamentos de rebeldia, e menos os de busca de emoção. No caso das condutas anti-sociais, 0 fator neuroticismo foi o único dos Cinco Grandes a explicá-las satisfatoriamente $\beta=-0,15, p<0,01) ; 0$ fator de extroversão logrou explicar a busca de emoção $(\beta=0,23, p<0,001)$. Os resultados a respeito do fator abertura à mudança, presente entre os Big Five, não aparecem como relevantes nos estudos prévios que 0 consideraram. Não obstante, Gullone e Moore (2000) observaram algumas correlações com comportamentos socialmente desviantes, como rebeldia $(r=-0,11, p<0,05)$ e imprudência $(r=-0,15, p<0,05)$. Porém, embora positiva, sua correlação foi fraca com a medida empregada de conduta anti-social $(r=0,05, p>0,05)$.

Percebe-se, pois, a relevância dos traços de personalidade delineados no modelo dos Big-Five e 0 papel do traço busca desensações como construto que pode contribuir para a compreensão das interações sociais, descrição e explicação dos comportamentos individuais (Heaven, 1996; Heaven, Caputi, Trivellion-Scott \& Swinton, 2000). Deste modo, verificou-se a influência de tais construtos na explicação das condutas desviantes. Para tanto, foi considerado o modelo dos Cinco Grandes Fatores enfocando, especificamente, os traços neuroticismo e extroversão, além do traço busca de sensações, como um fator geral.

\section{Método}

Neste estudo, assumiu-se um delineamento correlacional, expost facto, considerando como variável antecedente os traços de personalidade (neuroticismo, extroversão e busca de sensações) e, como critério, as condutas desviantes. Levando isto em conta, foram formuladas as seguintes hipóteses: 1) o traço de personalidade neuroticismo explicará diretamente as condutas anti-sociais; 2) o traço de personalidade extroversão explicará diretamente as condutas anti-sociais; 3) o fator geral de busca de sensações explicará diretamente as condutas anti-sociais; 4) as condutas anti-sociais predirão diretamente as condutas delitivas.

Participaram do estudo a que se refere este artigo 755 estudantes do Ensino Médio e Superior. Do total de participantes, 401 (53\%) eram do sistema privado e 354 (47\%) do sistema público da cidade de J oão Pessoa (PB), sendo $50,3 \%$ do sexo feminino e com idades varian-do entre 16 e 26 anos (média $(M)=20,21$; desvio-padrão (DP) $=3,12$ ).

Instrumentos

Os participantes responderam a um questionário composto por três medidas:

Escala de Condutas Anti-Sociais e Delitivas (CAD). Este instrumento, proposto por Seisdedos (1988), compreende uma medida comportamental composta por 40 itens (que expressam condutas desviantes), distribuídos em dois fatores, como segue: 1) Condutas Anti-sociais, cujos elementos não expressam delitos, mas comportamentos que desafiam a ordem social e infringem normas (por exemplo: jogar lixo no chão, mesmo quando há perto um cesto de lixo; tocar a campainha na casa de alguém e sair correndo); e 2) CondutasDelitivas. seus itens incorporam comportamentos delitivos que estão fora da lei, caracterizando uma infração ou uma conduta faltosa e prejudicial a alguém, ou mesmo à sociedade como um todo (por exemplo: roubar coisas de um lugar público; conseguir dinheiro ameaçando pessoas mais fracas). Para cada item, os participantes devem indicar com que freqüência apresentam 0 comportamento no seu dia a dia, utilizando uma escala de resposta com onze pontos, tendo os seguintes 
extremos: $0=$ nunca e $10=$ sempre Formiga e Gouveia (2003) adaptaram este instrumento, o qual apresentou parâmetros psicométricos satisfatórios; observaram-se índices de consistência interna (Alfa de Cronbach) de 0,91 para as condutas anti-sociais, $\mathrm{e} 0,86$ para as delitivas. Usando análise fatorial confirmatória, este autor comprovou igualmente a adequação desta estrutura $\left(\chi^{2} / \mathrm{g} . \mathrm{l}=1,78 ; \mathrm{GFI}=0,91, \mathrm{AGFI}=0,90\right.$ e RMSEA $\left.=0,04\right)$.

Inventário dos Cinco Grande Fatores da Personalidade. Elaborado porJ ohn, Donahue e Kentle (1991), também foi adaptado, mas por Gouveia et al. (2001). Utiliza-se aqui uma versão reduzida, composta por 14 itens, sendo sete para cada um dos dois fatores de interesse: extroversão (é sociável; extrovertido) e neuroticismo (fica nervoso facilmente; preocupa-se muito com tudo). Estesitens são respondidos em escala de cinco pontos, tipo Likert, com os seguintes extremos: $1=$ discordo totalmente $5=$ concordo totalmente Os índices de consistência intema (Alfa de Cronbach) desta medida ficaram acima de 0,70 , como segue: neuroticismo $(0,75)$ e extroversão $(0,74)$.

Escala deBusca deSensações (EBS). Construída por Zuckerman, Eysenck e Eysenck (1978), a versão empregada é composta de 23 itens, distribuídos em doisfatores: busca de aventura e emoção (por exemplo, gostaria de escalar uma montanha, ou gostaria de saltar de pára-quedas) e busca de experiência e excitação (por exemplo, sinto-me melhor depois de tomar alguns copos de bebida alcoólica, ou gostaria de provar drogas que causam alucinações). Estes itens são respondidos em escalas de cinco pontos, variando de $1=$ discordo totalmente a $5=$ concordo totalmente. Em uma ampla amostra (>1,000 participantes) na cidade de João Pessoa (PB), Vasconcelos (2004) comprova que os dois fatores desta medida estão diretamente correlacionados entre si $(r=0,38, p<0,001)$, sugerindo que pode ser considerado satisfatório o índice de consistência interna geral da escala (Alfa de Cronbach de 0,87).

Além das medidas listadas, os participantes responderam a algumas perguntas de caráter sócio-demográfico (por exemplo, sexo, idade, em que medida se consideram bons estudantes, entre outras). Estas contribuem para descrevê-los, assim como permitem, no caso de ser necessário, seu controle estatístico.
Procedimentos

Inicialmente, as instituições de ensino foram contatadas com a finalidade de solicitar a permissão para a realização da pesquisa. Em seguida, solicitou-se aos participantes sua colaboração para responder aos instrumentos. Estes foram aplicados de forma coletiva, em salas de aula, mas respondidos individualmente. A todos foi informado o objetivo geral do estudo e 0 caráter confidencial e sigiloso de suas respostas. Com o fim de minimizar os vieses de resposta, os instrumentos foram contrabalanceados, porém, deixando-se invariavelmente a folha de dados sócio-demográficos no final do questionário. Em média, foram necessários 40 minutos para o preenchimento total dos questionários.

Análise dos dados

A versão 11 do pacote estatístico SPSSWIN (Statistical Package for the Social Sciences) foi utilizada para tabulação e análise dos dados. Fez-se uso do programa AMOS 4 (Analysis Moment Structures) para a realização da análise do modelo proposto.

Indicadores estatísticos para o Modelo de Equações Estruturais (SEM) foram considerados segundo uma bondade de ajuste subjetiva, dada pelo $\chi^{2} / g l$ (grau de liberdade), que admite como adequados índices entre 2 e 3, aceitando-se até 5 ; índices de qualidade de ajuste, dados pelos $\mathrm{GFI} / \mathrm{AGFI}$, que medem a variabilidade explicada pelo modelo, e com índices aceitáveis a partir de 0,80; e a Raiz Quadrada Média Residual (RMR), que indica o ajustamento do modelo teórico aos dados, na medida em que a diferença entre os dois se aproxima de zero (Joreskög \& Sörbom, 1989).

\section{Resultados}

Tendo assegurado o procedimento de eliminação dos missings cases, optou-se por uma observação dos dados nas análises descritivas. Assim, a fim de caracterizar os participantes nas cond utasanti-sociais e delitivas, foram verificadas suas pontuações médias em relação à escala de resposta. Em um intervalo de variação de $0=$ nunca a $10=$ sempre, que avalia a freqüência de apresentação destas condutas, verificou-se que a média empírica nas condutas anti-sociais 
foi de 1,78 ( $D P=1,23$; amplitude mínima de zero e máxima de sete), enquanto nas condutas delitivas os participantes apresentaram uma média de 0,25 ( $D P=0,30$; e amplitude de zero a dois). Ambas as médias são estatisticamente inferiores ao ponto mediano teórico da escala de resposta ( $M d=5), t(754) \geq 71,96, p<0,001$.

Em seguida, visando averiguar se existem diferenças em relação ao gênero e à faixa-etária no que se refere às condutas anti-sociais e delitivas, foram realizadas análises de comparações de médias, especificamente Análise Multivariada de Variância (MANOVA).

Não foi observado efeito de interação das variáveis sexo e faixa etária dos participantes, porém, estas apresentaram efeito principal, Lambda deWilks de $0,91[F=35,01, p<0,001]$. Concretamente, com relação às condutas anti-sociais, verificou-se que os participantes do sexo masculino apresentaram média superior $(M=1,96, D P=1,33)$ à do grupo de mulheres $(M=1,60$, $\mathrm{DP}=1,09$ ), sendo esta diferença estatisticamente significativa [ $F=15,89, p<0,001]$. Quanto à faixa-etária, esta também se mostrou relevante; aqueles com idades entre 16 e 20 anos apresentaram média superior de condutas anti-sociais ( $M=1,90, D P=1,25)$ do que o fizeram os com idades de 21 a 26 anos ( $M=1,63, D P=1,20)[F=8,92, p<0,01]$. No caso das condutasdelitivas, unicamente se observou efeito principal da variável sexo $[F=68,66, p<0,001]$, com os homens apresentando média $(M=0,36, D P=0,42)$ superior à das mulheres $(M=0,14, D P=0,27)$.

\section{Testagem do Modelo}

A fim de lograr o objetivo central deste artigo, lembrando, testar o modelo teórico (causal) para explicar as condutas desviantes a partir dos traços de personalidade (busca de sensações, extroversão eneuroticismo), considerou-se um modelo recursivo de equações estruturais. Os pesos (saturações) que explicam o modelo proposto são expostos na Figura 1.

Como é possível observar na figura acima, após as devidas modificações encontrou-se um modelo adequado, apresentando uma razão $\chi^{2} / \mathrm{gl}=2,18$, com $\mathrm{GFI}=0,99, \mathrm{AGFI}=0,98, \mathrm{RMR}=0,010$ e RMSEA $=0,040$. Foi observado que os pesos das variáveis consideradas nos

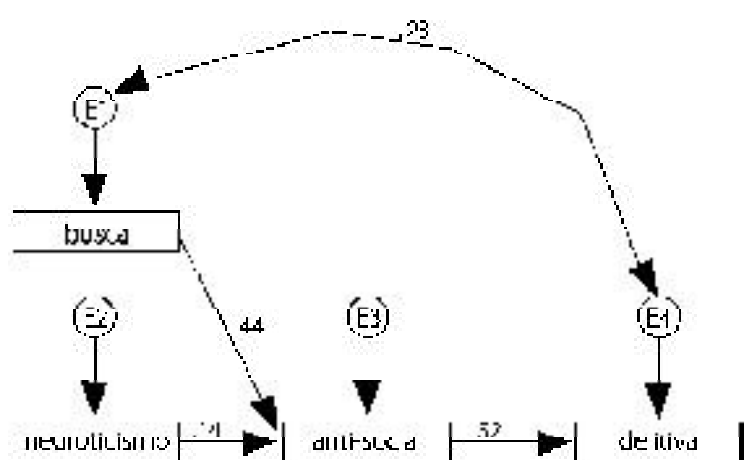

Figura 1. Modelo teórico paraexplicação das condutas desviantes pelos traços de personalidade.

direta e significativamente as condutas anti-sociais. Entretanto, o traço extroversão não se presentou significativo na explicação de tais condutas. As condutas anti-sociais também explicaram direta e significativamente as delitivas, como o esperado. Esta última foi influenciada pelo fator busca de sensações. Assim, foi estabelecida uma explicação para as condutas desviantes (anti-sociais e delitivas), a partir de dois fatores (busca de sensações e neuroticismo).

\section{Discussão}

Os objetivos deste estudo foram cumpridos. Visava-se, primordialmente, abordar o fenômeno das condutas socialmente desviantes apresentadas pelos adolescentes e jovens. Por ser este um aspecto deveras presente na sociedade contemporânea, muitos estudiosos têm se dedicado a analisá-lo (Adalbjarnardotti \& Rafnsson, 2002; Engels \& Bogt, 2001; Hawkins et al., 1992; Herrenkohl et al., 2000). Primeiramente, deve-se ter em mente que $o$ estudo das condutas anti-sociais e, em grau mais elevado, das condutas delitivas, apresenta algumas dificuldades, principalmente pela natureza complexa do fenômeno.

As condutas desviantes são consensualmente consideradas um problema multidimensional, e seus fatores norteadores podem se apresentar como os mais díspares possíveis. Pode-se mencionar a influência de fatores estruturais, culturais, institucionais, políticos, econômicos, sociais, históricos, psicológicos, biológicos, entre outros. Daí decorre a existência de pesquisadores das mais diversas áreas de conhecimento que se 
interessam pela temática. Frente ao exposto, decidiu-se aqui verificar em que medida, especificamente, os traços de personalidade (neuroticismo, extroversão e busca de sensações) são úteis para explicar as condutas anti-sociais e delitivas em um grupo de pessoas que têm características particulares: o grupo de adolescentes e jovens. Pfromm Neto (1979) coloca que muitas das transgressões dos adolescentes são, por vezes, uma ameaça à sociedade, porém, estas são freqüentemente estimuladas e consideradas necessárias para que 0 sujeito se sinta integrado ao seu grupo.

Em relação aos resultados expostos, como antes foram descritos, os jovens deste estudo apresentaram pontuações baixas nas medidas de condutas desviantes. 0 próprio fato de terem ingressado em escolas de ensino médio ou universidades torna menos provável que estes jovens se envolvam em condutas desta natureza. Apesar de estatisticamente representativa, em números, a amostra em questão tem um claro viés. Especificamente, considera apenas jovens que estão estudando. Ter uma atividade regular de aprendizagem tem sido mencionado como um elemento que inibe as condutas desviantes (Coelho Júnior, 2001; Hawkins et al., 1992). Além disso, os participantes deste estudo, embora possam apresentar comportamentos desta natureza, não têm exatamente um histórico de delinqüência. Tratam-se, pois, de jovens da população geral dos estudantes. Este aspecto se deixa transparecer quando são consideradas as pontuações médias que estes apresentaram nas medidas de condutas desviantes - claramente, muito abaixo do ponto mediano da escala.

No que tange às possíveis diferenças em relação ao sexo e à faixa-etária, coerentemente com os estudos prévios, os jovens diferiram significativamente em relação às condutas desviantes em função da faixa etária. Os homens, e com idades entre 16 e 20 anos, são mais sujeitos a apresentar estas condutas (Formiga, 2002). Esta diferença entre homens e mulheres provavelmente ocorre devido aos fatores envolvidos no processo de socialização, visto que a menina é educada para 0 diálogo, enquanto o menino é educado para a ação. No caso da idade, os jovens de 16 a 20 anos se encontram em plena adolescência, conforme a OMS (Bahls \& Ingbermann, 2005), sendo este um período em que é alta a probabilidade de se querer provar coisas, buscar aventuras, realizar travessurase romper normas (Coelho Júnior, 2001; Petraits, Flay \& Miller, 1995).

As principais indicações do modelo proposto revelam o poder de explicação dos traços de personalidade. Observou-se que os traços neuroticismo e busca de sensações foram os únicos no modelo que predizem satisfatoriamente as condutas anti-sociais de forma direta; neste sentido, o traço extroversão não colaborou na explicação de tais condutas. As pessoas que apresentam alta pontuação no traço neuroticismo são, geralmente, ansiosas, tensas e emotivas. Têm alta probabilidade de apresentarem problemas na sua auto-estima. De acordo com Benet-Martínez e John (1998), este traço põe em destaque os afetos negativos dos sujeitos, os quais incluem tristeza, irritabilidade e tensão nervosa. Daí, pode se verificar a sua relevância para explicar as condutas em questão, pois, muitas vezes, as pessoas, por apresentarem estas características, diferenciam-se do padrão de comportamento que é socialmente esperado, podendo, então, ser percebidas e perceberem a si mesmas como anti-sociais.

o segundo traço que se mostrou relevante no modelo foi a busca de sensações. Como dito, este reflete a característica de querer buscar novas e intensas experiências. No modelo testado, o traço explicou diretamente as condutas anti-sociais, sendo o traço com maior peso fatorial no modelo. Estes achados corroboram os estudos que tratam da relação entre a busca de sensações e os comportamentos de risco. A despeito, Lin e Tsai (2002) encontraram que tanto comportamentos de risco socialmente aceitáveis (como é o caso de pára-quedismo e atividades de mergulho), quanto os não aceitáveis (por exemplo, dirigir embriagado e usar drogas) estão diretamente relacionados a altas pontuações em busca de sensações.

Estes achados confirmam o que aponta Zuckerman (1983; 1994) acerca dos altos buscadores de sensações. Para este autor, eles têm algo de anti-social em seu senso, o que faz com que seus comportamentos estejam baseados em sua própria vontade, ao invés das convenções sociais ou das vontades de outrem. Ademais, eles são não-conformistas e valorizam situações de risco.

Em resumo, os traços de personalidade, a exemplo dos cinco grandes fatores, têm sido considerados como construtos importantes para explicar as 
condutas anti-sociais e delitivas (Heaven, 1996). Contudo, o traço busca de sensações parece ser mais relevante neste contexto, uma vez que revela padrões de condutas intimamente relacionados com a possibilidade de assumir riscos.

Finalmente, cabe destacar a necessidade de novos estudos que abordem a temática em questão. Neste sentido, é importante considerar outras variáveis apontadas pela literatura como úteis à análise deste fenômeno no qual, cada vez mais, estão envolvidos crianças, adolescentes, jovens e adultos, atingindo diversas classes sociais e níveis de escolaridade.

\section{Referências}

Adalbjarnardottir, S., \& Rafnsson, F. D. (2002). Adolescent antisocial behavior and substance use longitudinal analyses. AddictiveBehaviors, 27 (2), 227-240.

Allport, G. W. (1973). Personalidade: padrões e desenvolvimento. São Paulo: EPU.

Aluja, A. (1989). El rasgo de personalidad "Búsqueda de Sensaciones": una teoría psicobiológica. Cuadernos de Medicina Psicosomática y Sexología, 11/12,59-81.

Aluja, A. (1990). La teoría de la "Búsqueda de Sensaciones" y la teoría de la personalidad de Eysenck: puntos de contacto. CuadernosdeM Medicina Psicosomática ySexología, 16, 43-50.

Bahls, F. R. C., \& Ingbermann, Y. K. (2005). Desenvolvimento escolar e uso de drogas na adolescência. Estudos de Psicologia (Campinas), 22 (4), 395-402.

Benet-Martínez, V., \& John, O. P. (1998). Los cinco grandes across cultures and ethnic groups:multitrait-multimethod analyses of the Big Five in Spanish and English.J ournal of Personality and Social Psychology, 75 (3), 729-750.

Cattell, H. E. P. (1996). The original Big Five: a historical perspective. European Review of Applied Psychology, 46 (11), 5-14.

Cattell, R. B., \& Cattell, H. E. P. (1995). Personality structure and the new fifth edition of the 16PF. Educational and Psychological Measurement, 55 (6), 926-937.

Coelho Júnior, L. L. (2001). Uso potencial de drogas em estudantes do ensino médio: suas correla ções com as prioridades axiológicas. Dissertação de mestradonão- publicada. Departamento de Psicologia, Universidade Federal da Paraíba, João Pessoa.

Costa, P. T., \& McCrae, R. R. (1992). The five-factor model of personality and its relevance to personality disorders. J ournal of Personality Disorders, 6 (4), 343-359.

Digman, J. M. (1990). Personality structure: emergence of the five-factor model. In M. R. Rosenzweig \& L. W. Porter (Eds.), Annual review of psychology (Vol.41, pp.417-440).
Ding, C. S., Nelsen, E. A., \& Lassonde, C. T. (2002). Correlates of gun involvement and aggressiveness among adolescents. Youth and Society, 34 (2), 195-213.

Donohew, R. L., Hoyle, R. H., Clayton, R. R., Skinner, W. F., Colon, S. E., \& Rice, R. E. (1999). Sensation seeking and drug use by adolescents and their friends: models for marijuana and alcohol. Journal Study of Alcohol, 60 (5), 622-631.

Engels, R. C. M., \&Bogt, T. (2001). Influences of risk behaviors on the quality of peer relations in adolescence. Journal of Youth and Adolescence, 30 (6), 675-695.

Eysenck, H. J. (1990). Genetic and environmental contributions to individual differences: the three major dimensions of personality. J ournal of Personality, 58 (1), 245-261.

Eysenck, H. J. (1991). Dimensions of personality: 16, 5, or 3 ? Criteria for a taxonomic paradigm. Personality and Individual Differences, 12 (8), 773-790.

Feingold, A. (1994). Gender differences in personality: a meta-analysis. Psychological Bulletin, 116 (3), 429-456.

Five Factor Model. (2001).Greatideasin personality:fivefactor model. Retrieved May 4, from http//www.personality research. org/bigfive.html

Formiga, N. S. (2002). Condutas anti-sociais e delitivas: uma explicação baseada nos valores humanos. Dissertação de mestrado não-publicada, Departamento de Psicologia, Universidade Federal da Paraíba, J oão Pessoa.

Formiga, N. S., \&Gouveia, V. V. (2003). Adaptação e validação da escala de condutas anti-sociais e delitivas ao contexto brasileiro. Psico, 34(2), 367-388.

Goldberg, L. R. (1981). Language and individual differences: the search for universals in personality lexicons. In L. Wheeler (Ed.), Review of personality and social psychology (Vol.2, pp.141-165). Beverly Hills, CA: Sage.

Gouveia, V. V., Coelho Júnior, L. L., Gontiès, B., Andrade. J. M., $\&$ Andrade, P. R. (2003). Fatores de risco para o uso de drogas entre adolescentes. Thomson Psicología, 1 (1), 135-51.

Gouveia, V. V., Meira, M., Santos, W. S., J esus, G. R., \& Formiga, N. S. (2001). Personalidade e valores humanos. Trabalho apresentado no II Congresso Norte Nordeste de Psicologia, Salvador, BA.

Greene, K., Krcmar, M., Walters, L. H., Rubin, D. L., \& Hale, L. (2000). Targeting adolescent risk-taking behaviors: the contributions of egocentrism and sensation-seeking. Journal of Adolescence, 23 (4), 439-461.

Gullone, E., \& Moore, S. (2000). Adolescent risk-taking and the five-factor model of personality.J ournal ofAdolescent, 23 (4), 393-407.

Hall, C. S., Lindzey, G., \& Campbell, J. B. (2000). Teorias da personalidade. Porto Alegre, ArtMed.

Hawkins, J. D., Catalano, R. F., \& Miller, J. Y. (1992). Risk and protective factors for alcohol and other drug problems in adolescent and early adulthood: implications for substance abuse prevention. Psychological Bulletin, 112 (1), 64-105. 
Heaven, P. C. L. (1994). Family of origin, personality, and self-reported delinquency. Journal of Adolescence, 17 (5), 445-459.

Heaven, P. C. L. (1996). Personality and self-reported delinquency: analysis of the "big five" personality dimensions. Personality and Individual Differences, 20 (1), 47-54.

Heaven, P. C. L., Caputi, P., Trivellion-Scott, D., \& Swinton, T. (2000). Personality and group influenceson self-reported delinquent behavior. Personalityand Individual Differences, 28 (6), 1143-1158.

Herrenkohl, T. I., Maguin, E., Hill, K. G., Hawkins, J . D., Abbott, R. D., \& Catalano, R. F. (2000). Developmental risk factors for youth violence. Journal of Adolescent Health, 26 (3), 176-186.

Hofstede, W. K. B., Raad, B., \& Goldberg, L. R. (1992). Integration of the Big Five and circumplex approaches to trait structure. Journal of Personality and Social Psychology, 63 (1), 146-163.

Hutz, C. S., Nunes, C. H., Silveira, A. D., Serra, J., Anton, M., \& Wieczorek, L. S. (1998). O desenvolvimento de marcadores para a avaliação da personalidade no modelo dos cinco grandes fatores. Psicologia:Reflexão e Crítica, 11 (2), 395-410.

J esus, G. R. (2001). Personalidadee gênero: sua relação com os valores humanos básicos. Monografia não-publicada, Departamento de Psicologia, Universidade Federal da Paraíba, João Pessoa.

Joreskög, K., \& Sörbom, D. (1989). LISREL 7 user's reference guide. Mooresville: Scientific Software.

Katigbak, M. S., Church, A. T., Cuazon-Lapefia, M. A., Carlota, A. . ., \& Del Pilar, G. H. (2002). Are indigenous personality culture-specific? Philippine inventories and the five-factor model. Journal of Personality and Social Psychology, 82 (1), 89-101.

Kopeikin, H. S. (1997). Sensation seeking. Retrieved May 15 , 2002, from http://www.psych.ucsb.edu/ kopeikin sssinfo.htm

Laak, J. T. (1996). Las cinco grandes dimensiones de la personalidad. Revista de Psicología de la PUCP, 14 (2), 129-181.

Lin, S. S. J ., \& Tsai, C. (2002). Sensation seeking and Internet dependence of Taiwanese school adolescents. Computers in Human Behavior, 18 (4), 411-426.

McCrae, R. R., \& John, O. P. (1992). An introduction to the Five-factor model and its applications. Journal of Personality, 60 (2), 1 75-216.

Patterson, G. R., DeBaryshe, B. D., \& Ramsey, E. (1989). A developmental perspective on antisocial behavior. American Psychologist, 44 (4), 329-335.

Paunonen, S. V., \& Ashton, M. C. (2001). Big five and facets the prediction of behavior. Journal of Personality and Social Psychology, 74 (2), 538-556.

Perugini, M., Gallucii, M., \& Livi, S. (2000). Looking for a simple Big Five factorial structure in the domain of
Adjectives. European J ournal of Psychological assessment 16 (2), 87-97.

Pervin, L.A. (1978). Personalidade:teoria, avaliação epesquisa. São Paulo: EPU.

Petraits, J., Flay, B. R., \& Miller, T. Q. (1995). Reviewing theories of adolescent substance use: organizing pieces in the puzzle. Psychological Bulletin, 117 (1), 67-86.

Pfromm Neto, S. (1979). Psicologia da adolescência. São Paulo: Pioneira.

Pimentel, C. E. (2004). Valores humanos, preferência musical, identificação grupal e comportamentos anti-sociais. Dissertação de mestrado não-publicada, Departamento de Psicologia, Universidade Federal da Paraíba, João Pessoa.

Queiroga, F. (2002). 0 modelo dos cinco grandes fatores da personalidade: sua relação com as prioridades valorativase variáveis sócio-demográficas. Relatório de Pesquisa. Departamento de Psicologia, Universidade Federal da Paraíba, João Pessoa.

Romero, E., Luengo, M. A., \& Sobral, J. (2001). Personality and antisocial behavior: study of temperamental dimensions. Personality and Individual Differences, 31 (3), 329-348.

Saner, H., \& Ellickson, P. (1996). Concurrent risk factors for adolescence violence. J ournal of Adolescent Health, 19 (2), 94-103.

Scaramella, L. V., Conger, R. D., Spoth, R., \& Simons, R. L. (2002). Evaluation of a social contextual model of delinquency: a cross-study replication.Child Development, 73 (1), 175-195.

Schultz, D. P., \& Schultz, S. E. (2002). Teoriasda personalidade. São Paulo: Pioneira Thomson.

Seisdedos, N. C. (1988). Cuestionario A - D de conductas antisociales- delictivas. Madri: TEA.

Vasconcelos, T. C. (2004). Personalidade, valores e condutas anti-sociais de jovens. Dissertação de mestrado nãopublicada. Universidade Federal da Paraíba,J oão Pessoa.

Waiselfisz, J. J. (2002). Mapa da violência III: osjovens do Brasil. Brasília: UNESCO.

Zuckerman, M. (1983). A biological theory of sensation seeking. In M.Zuckerman (Ed.),Biological basesof sensation seeking, impulsivity and anxiety. Hillsdale, NJ: Lawrence Erlbaum Assoc.

Zuckerman, M. (1994). Behavioral expressions and biosocial bases of personality. New York: Cambridge University Press.

Zuckerman, M., Eysenck, S. B. G., \& Eysenck, H. J. (1978). Sensation seeking in England and America:cross-cultural, age and sex comparisons. Journal of Consulting Clinical Psychology, 46 (1), 139-149.

Recebido em:27/7/2006

Versão final reapresentada em:29/1/2007

Aprovado em:23/4/2007 
